\title{
Crystal Fields and the Magnetic Properties of Praseodymium and Neodymium
}

\author{
Johansson, Torben; Lebech, Bente; Nielsen, Mourits; Bjerrum Møller, Hans; Mackintosh, Allan
}

\section{Published in:}

Physical Review Letters

Link to article, DOI:

10.1103/PhysRevLett.25.524

Publication date:

1970

Document Version

Publisher's PDF, also known as Version of record

Link back to DTU Orbit

Citation (APA):

Johansson, T., Lebech, B., Nielsen, M., Bjerrum Møller, H., \& Mackintosh, A. (1970). Crystal Fields and the Magnetic Properties of Praseodymium and Neodymium. Physical Review Letters, 25(8), 524-526.

https://doi.org/10.1103/PhysRevLett.25.524

\section{General rights}

Copyright and moral rights for the publications made accessible in the public portal are retained by the authors and/or other copyright owners and it is a condition of accessing publications that users recognise and abide by the legal requirements associated with these rights.

- Users may download and print one copy of any publication from the public portal for the purpose of private study or research.

- You may not further distribute the material or use it for any profit-making activity or commercial gain

- You may freely distribute the URL identifying the publication in the public portal 
Phys. Rev. B 1, 3250 (1970), Appendix B.

${ }^{16}$ S. R. Broadbent and J. M. Hammersley, Proc. Cambridge Phil. Soc. 53, 629 (1957).
${ }^{17}$ H. L。 Frisch, J. M. Hammersley, and D. J.A. Welsh, Phys. Rev. 126, 949 (1962).

${ }^{18} \mathrm{~K}$. Freed and M. H. Cohen, to be publsihed.

\title{
CRYSTAL FIELDS AND THE MAGNETIC PROPERTIES OF PRASEODYMIUM AND NEODYMIUM
}

\author{
T. Johansson \\ Technical University, Lyngby, Denmark
}

and

B. Lebech, M. Nielsen, and H. Bjerrum Møller

Atomenergikommissionen Research Establishment, Ris $\phi$, Denmark

and

A. R. Mackintosh

H. C. Ørsted Institute, University of Copenhagen, Denmark

(Received 29 June 1970)

\begin{abstract}
The magnetic properties of $\operatorname{Pr}$ and Nd single crystals have been studied by neutrondiffraction and susceptibility measurements. In contrast to earlier results on polycrystals, monocrystalline $\mathrm{Pr}$ is found not to be magnetically ordered, because of crystal field effects, but a magnetic field induces a large moment. Anisotropic effective exchange results in a large magnetic anisotropy. The complex magnetic structure of $\mathrm{Nd}$ is substantially modified by a magnetic field.
\end{abstract}

The magnetic properties of the light rareearth metals $\operatorname{Pr}$ and Nd are of particular interest because the crystal-field splittings of the magnetic energy levels in the double-hexagonalclose-packed (dhcp) structure are comparable with the exchange energies. This is in contrast to, for instance, the heavy rare earths, where the crystal field acts as a source of magnetic anisotropy which, though relatively strong, is still small compared with the exchange. We have investigated these crystal-field effects in single crystals by neutron-diffraction experiments in fields as large as $50 \mathrm{kG}$, and through magnetic susceptibility measurements by the Faraday method.

The dhcp structure consists of two inequivalent sets of ionic sites, one of which is in a local environment with hexagonal symmetry and the other with cubic symmetry. The crystal-field energy levels in $\operatorname{Pr}$ have been considered by Bleaney ${ }^{1}$ who showed that the ground states of the ions at both types of site are singlets; thus, magnetic ordering will not occur unless the ratio of exchange to crystal-field interactions exceeds a critical value. ${ }^{2}$ On the assumption that no magnetic ordering actually occurs in $\mathrm{Pr}$, he calculated a number of properties, including the magnetic susceptibility. Systems with crystal-field singlet ground states have been extensively discussed by Wang and Cooper. ${ }^{3}$ Cable et al. ${ }^{4}$ showed by neutron diffraction that a polycrystalline sample of $\mathrm{Pr}$ was antiferromagnetic with a Néel temperature of about $25^{\circ} \mathrm{K}$, and suggested that only the hexagonal sites order. We observed no trace of spontaneous magnetic ordering in a single crystal of $\operatorname{Pr}$ at $4.2^{\circ} \mathrm{K}$. Since the occurrence of antiferromagnetism in a polycrystalline sample and its absence in a monocrystal has also been observed by Rainford and Wedgwood, ${ }^{5}$ we conclude that pure monocrystalline $\mathrm{Pr}$ is not antiferromagnetic but that the exchange is sufficiently great that a small modification of the crystal-field splittings, perhaps due to strains, can lead to spontaneous ordering.

The application of a magnetic field along the $\vec{b}_{2}$ (110) direction produces a large induced moment, which shows a substantial tendency towards saturation at high fields and low temperatures, as shown in Fig. 1. By observing the neutron-diffraction intensities at different reciprocal-lattice points, it is possible to separate the contributions from the cubic and hexagonal sites; we find that $\mu$ (hexagonal $)=1.8 \mu_{\mathrm{B}} /$ ion while $\mu$ (cubic) $=0.9 \mu_{\mathrm{B}} /$ ion at $4.2^{\circ} \mathrm{K}$ and $46 \mathrm{kG}$. A fairly good fit to these results may be obtained ${ }^{5}$ using a molecular-field model and the crystal-field level scheme deduced by Bleaney, ${ }^{1}$ except that the best value for the energy separation between the ground state and the first-excited doublet 

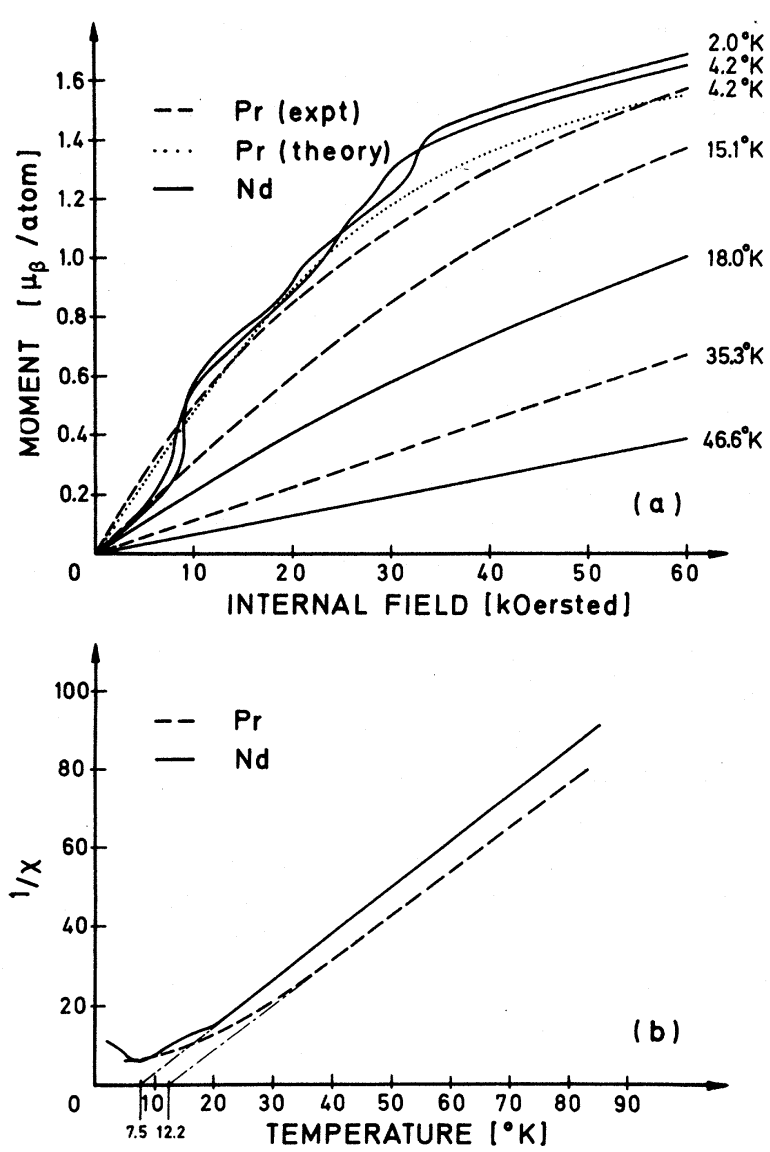

FIG. 1. (a) The variation of moment with internal magnetic field applied in the (110) direction in Pr and Nd. For clarity the experimental points have been omitted; the measurements were performed with a relative accuracy of better than $1 \%$. The dotted curve is a calculation of Rainford, Ref. 5, based on the model of Bleaney, Ref. 1, as discussed in the text. (b) The low-field susceptibility of $\mathrm{Pr}$ and $\mathrm{Nd}$ in the (110) direction as a function of temperature. The anomalies in the susceptibility of Nd occur at the magnetic transition temperatures.

on the hexagonal sites is found to be about $20^{\circ} \mathrm{K}$, considerably smaller than Bleaney's value of $63^{\circ} \mathrm{K}$. The molecular-field constant is positive and close to the critical value necessary for the occurrence of ferromagnetism. Preliminary neutron-diffraction and magnetic-susceptibility measurements with the field in the $\vec{b}_{3}(001) \mathrm{di}-$ rection reveal that the susceptibility is an order of magnitude smaller than that in the basal plane. A large susceptibility anisotropy was predicted by Bleaney, ${ }^{1}$ but in the opposite sense to that observed. In the absence of exchange effects, he found $\chi\left(\vec{b}_{3}\right)$ to be about 3 times greater than $\chi\left(\vec{b}_{2}\right)$. The striking reversal of this anisotropy requires an anisotropic molecular-field constant, which must take a large negative value when the field is in the $\vec{b}_{3}$ direction, to account for the low susceptibility. An anisotropic effective exchange is one of the features of the Wang and Cooper pseudospin formulation of the singlet ground-state problem. ${ }^{3}$

$\mathrm{Nd}^{3+}$ is a Kramers ion and the crystal-field levels are, therefore, at least doubly degenerate. The magnetic structure in zero field has been studied by Moon, Cable, and Koehler. ${ }^{6}$ Our observations are in substantial agreement with theirs. At $19.2^{\circ} \mathrm{K}$ the moments on the hexagonal sites form a periodic structure,

$$
\vec{\mu}_{B}=-\vec{\mu}_{C}=\mu_{h} \hat{b}_{1} \cos \left(\vec{Q}_{h} \cdot \vec{R}\right),
$$

where $B$ and $C$ refer to layers of ions in the $A B A C$ dhcp structure. The ordered moments lie along a $\vec{b}_{1}$ direction, as does the temperaturedependent wave vector, $\vec{Q}_{h}$. At $7.5^{\circ} \mathrm{K}$ the cubic sites order according to

$$
\vec{\mu}_{A}=-\vec{\mu}_{A^{\prime}}=\mu_{c} \hat{\mathrm{b}}_{2} \cos \left(\overrightarrow{\mathrm{Q}}_{c} \cdot \overrightarrow{\mathrm{R}}\right) \text {, }
$$

where $A$ and $A^{\prime}$ are neighboring cubic layers. The moments lie along $\vec{b}_{2}$ while $\vec{Q}_{c}$, which is again temperature dependent, is along $\vec{b}_{1}$. There is a slight dependence of the $\vec{Q}$ vectors on the relative orientations of the moments on the two sublattices in different domains. This effect may be seen as a splitting of the hexagonal satellites in Fig. 2, and also in a similar splitting of the cubic satellites at higher temperatures. Because of crystal-field effects, the maximum moment in zero field does not approach the theoretical maximum $g \mu_{\mathrm{B}} J=3.2 \mu_{\mathrm{B}}$ at low temperatures, on either type of site.

We have investigated the effect of a magnetic field in the $\vec{b}_{2}$ direction on these structures, again by a combination of neutron-diffraction and susceptibility experiments. Our results may be interpreted as follows: At $4.2^{\circ} \mathrm{K}$ a single-domain crystal is formed at about $7 \mathrm{kG}$, with the $\vec{Q}$ vectors normal to the field. In low fields the moments on the hexagonal sites turn parallel to those on the cubic sites; this process is complete at about $12 \mathrm{kG}$. At the same time a ferromagnetic moment is developed on the cubic and hexagonal sites in the ratio of approximately $5: 2$. At about $23 \mathrm{kG}$ there is an abrupt increase in both the ferromagnetic and periodic moments on the hexagonal sites so that the greatest total moment at a hexagonal site approaches the theoretical maximum. We tentatively ascribe this abrupt change to the crossing of crystal-field levels in the magnetic field. Above this field 


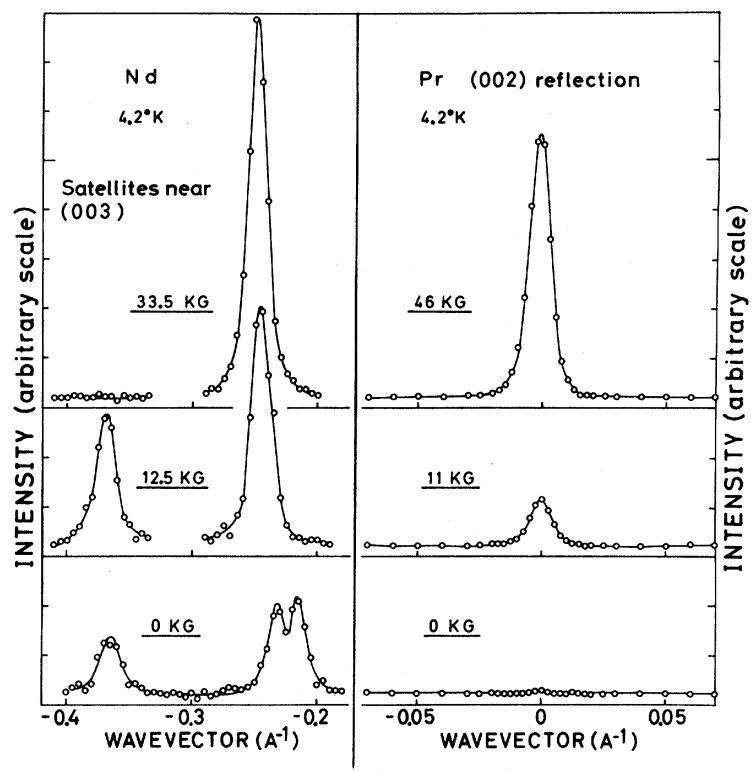

FIG. 2. Neutron diffraction patterns from Nd and Pr when a magnetic field is applied in the (110) direction. The larger peak in $\mathrm{Nd}$, split in zero field, originates from the moments on the hexagonal sublattice, and the smaller, from the cubic sublattice. In $\mathrm{Pr}$ the structure factor is such that the (002) reflection measures the difference between the moments on the two sublattices.

the ferromagnetic moment on the hexagonal sites grows at the expense of the periodic moment. The periodic moment on the cubic sites decreases steadily with field and disappears at about 31 $\mathrm{kG}$, with a corresponding increase in the ferromagnetic moment. Analogous changes in the moment distribution are produced by a field at other temperatures and are reflected in the magnetic moment measurements of Fig. 1, which agree with earlier low-field results. ${ }^{7}$ In contrast to the case of $\mathrm{Pr}$ there are clear anomalies in the low-field susceptibility measurements for $\mathrm{Nd}$, which occur at the magnetic transition temperatures.

We therefore conclude that, as expected, crystal-field effects are of crucial importance in determining the magnetic properties of $\mathrm{Pr}$ and $\mathrm{Nd}$. In $\mathrm{Pr}$ the crystal-field splittings from the singlet ground states are sufficient to inhibit magnetic ordering, although a magnetic field may induce a large moment. The very large magnetic anisotropy appears to be dominated by the anisotropy of the effective exchange. The crystal fields in $\mathrm{Nd}$ restrict the magnitude of the ordered moment in zero field, but the total ordered moment can be substantially increased by a magnetic field. Abrupt changes in the magnetization may be due to the crossing of crystal-field levels. Further investigations of these phenomena, both experimental and theor stical, are in progress.

We are gratesul to Dr. B. D. Rainford for discussing these experiments with us, and for informing us of his work before publication. Valuable discussions with Dr. B. R. Cooper are also gratefully acknowledged.

${ }^{1}$ B. Bleaney, Proc. Roy. Soc., Ser. A 276, 39 (1963).

${ }^{2}$ G. T. Trammell, J. Appl. Phys. 31, 362S (1960).

${ }^{3}$ Y. - L. Wang and B. R. Cooper, Phys. Rev. 172, 539 (1968), ibid. 185, 696 (1969).

${ }^{4} \mathrm{~J}$. W. Cable, R. M. Moon, W. C. Koehler, and E. O. Wollan, Phys. Rev. Lett. 12, 553 (1964).

${ }^{5}$ B. D. Rainford, private communication.

${ }^{6}$ R. M. Moon, J. W. Cable, and W. C. Koehler, J. Appl. Phys. 35, 1041 (1964) .

${ }^{7}$ D. R. Behrendt, S. Legvold, and F. H. Spedding, Phys. Rev. 106, 723 (1957). 